13. In this paper Professor Hedrick describes a method of characterizing functions of a complex variable that are not analytic, but that differ only slightly from analytic functions. For example, the function $\psi(z)=f(z)+\epsilon \varphi(z)$, where $f(z)$ is analytic, and $\epsilon$ is a real number, will differ but little from $f(z)$, if $\epsilon$ is chosen small, at all points where $|\varphi(z)|$ is finite. The Riemann surface for such functions may be studied by means of its relation to that for $f(z)$. A series of examples of this type and of more general types is given.

O. D. KellogG, Secretary of the Section.

\title{
THE RELATIONS OF MATHEMATICS TO THE NATURAL SCIENCES.
}

PRESIDENTIAL ADDRESS DELIVERED BEFORE THE AMERICAN MATHEMATICAL SOCIETY, DECEMBER 28, 1916.

BY PRESIDENT E. W. BROWN.

The duty enjoined on the President of the American Mathematical Society of delivering an address at the close of his term of office is at once an opportunity and a danger. It is one of the rare occasions when he is able to discuss matters which are unsuited for a memoir and when it is proper to try to take a somewhat broader view of his subject than is suggested in investigations designed to elucidate some special part of it. In so doing, he necessarily must look into the future and attempt to foresee it through such indications of the present as may seem significant; and the danger of becoming a false prophet or of raising an unnecessary alarm is unattractive to anyone, least of all to those who have the lifelong habit of feeling their way into the unknown by roads slowly constructed and securely laid. I am willing to run this risk because I believe that there are certain matters connected with the future of mathematical science which need fuller consideration than they have received of late years. In discussing them, one must necessarily tread on debatable ground. It is, however, a happy custom to regard the matter contained in a presidential address not as an official presen- 
tation of the views of the Society but solely as the personal opinion of the one member appointed to deliver it. Such suggestions as I have to make in connection with my topic "The Relations of Mathematics to the Natural Sciences" have no claim to novelty or originality. They are partly the natural result of association with those who work in more or less closely related fields and partly of a long connection with a task which has led in directions away from those usually followed by the modern mathematician. Before this discussion, however, a brief summary of the principal events of the past two years may not be out of place.

The summer meeting of the Society in Cambridge, as you are aware, was made the occasion for a celebration of our twenty-fifth anniversary. Originally started in 1888 as a meeting of those interested in mathematics in New York and its vicinity, the Society emerged three years later in the form of a national organization with a BULLETIN containing a record of the activities of its members and with some limited publication of their work and ideas. Nine years later, the growth of research in America, unquestionably assisted by the opportunities afforded by the Society, caused a need of further facilities for publication and the Transactions was started. Both journals have grown beyond the sizes originally contemplated and we may hope or fear that a new medium for publication may be required in the not distant future. The original meeting place in New York City has been quite insufficient to supply our members with opportunities to present papers and to engage in discussions; one after another, sections have been founded in various parts of the country which hold sessions at regular intervals and which assist actively in contributing to the growth of the subject and the welfare of the society. Our library numbers over 5,000 volumes, besides a large collection of unbound dissertations. And finally the offices of the Society and most of its records have survived the fire which seems to be a necessary preliminary to permanent stability.

This growth has lately caused a serious problem to arise in connection with the extent of the activities of the Society. Many of those who on account of professional duties or for other causes are unable to follow or lead far along the highroads of research, have felt that there are many byways in mathematics which are of interest and which would stimulate 
thought. They felt too that some centralization of efforts to improve the teaching of mathematics was necessary. The Society had hitherto confined its main activities to research and it became a grave question whether it should undertake new duties and responsibilities in addition to the already heavy burdens laid on certain of its officers. The difficulty has been happily solved by the formation of the Mathematical Association of America with assumption of responsibility for the American Mathematical Monthly and its use as the official journal of the Association. This result has been brought about by the cordial cooperation of everyone concerned. They have desired not only that the interests of the American Mathematical Society should not suffer but that the new Association should be so organized and conducted as to assist in strengthening and coordinating all forms of mathematical activity throughout the country. Our best wishes go out to our young and already vigorous offspring.

In outside activities, the Society during the last two years has appointed representatives to the general Committee on the classification of technical literature, to the Committee on standards for graphic representation and to the Naval Advisory Board. In spite of some misconception in the minds of those who were responsible for the invitation to the last named, as to the functions of mathematicians, both the Society and the Board are to be congratulated on the work of our two representatives.

Perhaps the most important outside movement affecting the chief objects of the Society is that initiated by the National Academy of Sciences for the organization and development of research. Originally started with the object of assisting in the defence of the country, it enlarged its functions when it was seen that much would be gained through cooperation and mutual assistance not only by investigators themselves but by all those who need and employ investigators in technical and manufacturing processes. This is therefore a movement in the interests of the whole community. The Society has already, through the Council, expressed its desire to cooperate. We may hope that there will result some benefits for our science and that we shall be able to furnish in turn some assistance toward the solution of the problems presented by other sciences.

With this record of achievement in the past it is but natural 
that we should look forward to the future and note whether, besides promise of progress and success, there are indications of danger against which measures of protection or alleviation are necessary.

Mathematics may be treated as a science or art which holds within itself all that is necessary for its own development. It may also be treated as an aid for the development of the natural sciences and for all forms of investigation which admit of deduction from exact statements. In the one, an advance in knowledge of the subject itself by examination of the interrelations of a set of axioms is sought; in the other, it is used to deduce phenomena of nature as the consequences of physical laws which have themselves been deduced by observation or experiment. In the earlier history of the subject, the difference between the two points of view was but little recognized. The axioms themselves were mainly suggested by natural phenomena and the developments were principally those needed in the elucidation of the phenomena. As time went on, mathematics was allowed to progress in its own way, unhindered by any necessity for present or future applications. This freedom from a somewhat entangling alliance has resulted in a marvellous structure of thought. The fundamental bases of the subject have been clarified and organized, the modes of reasoning have become subject to careful scrutiny and every effort has been directed to make sure that the structure built on these lines shall be without blemish.

There is, however, a reverse side to the picture. While every other product of human thought demands aid from outside, modern pure mathematics stands practically alone. Its laws, its logic, what it seeks and what it finds have no necessary relation to externals in the sense of dependence. And this independence has been achieved by no ordinary labor and by the exercise of many minds possessing gifts of insight in the highest degree. Yet one cannot help asking whether it is for the best interests of the subject that it should continue in this isolation. An external stimulus seems to be necessary, at least from time to time, to produce the best elements of growth in most forms of human activity. Is the continued development of pure mathematics an exception to the rule? One may argue that isolation produces a pure strain, but is it not also true that an occasional crossing of the breed is necessary to prevent the species from running itself 
out? I do not wish to imply that this is a real analogy; whether it is so or not, it can at least serve as an illustration of the manner in which the separation may affect mathematics. If it be admitted that future injury is likely to result, it is obvious that we should search for methods to alter the condition; if the contrary is true, I can still fall back upon the demand that other sciences make for assistance.

At the outset one must admit that the separation is not altogether confined to the relations of mathematics to the natural sciences, it has been growing even between its different branches, not so much perhaps in essentials as in the fact that there are only a few amongst us who can follow except in a dim way or with much labor, the larger part of the advances which are being published so frequently. This is partly due to the considerable body of knowledge which has accumulated: time and opportunity are lacking to assimilate even a small portion of it. And there are other contributing causes which will occur to you. Over one of them, of minor importance perhaps but in which some improvement may be possible, I shall delay for a moment.

The symbolism employed to convey ideas in mathematics is unquestionably good in its general outlines and serves the purposes of the study well. It is brief, it admits of considerable variety, and it is easily adapted to most of the needs of the mathematician. Unfortunately, few standards have been kept. While the general principles by which the notation is to be used are little changed, the widest variations in the meanings of different sets of symbols have been allowed to creep in; and conversely, the same sets of ideas have been frequently represented by a variety of symbols. In my own subject of celestial mechanics, this variety continually halts one's thoughts although the mathematical ideas are comparatively simple; in fact the symbols rarely represent anything else than the most elementary operators and quantities which are either fixed or have continuous variation. But the difficulty is far greater where the ideas themselves are complex, where a single symbol may imply numerous properties and where different authors adopt different meanings to be attached to the same symbol. The perfectly simple triad of marks " (1)," for example, may represent a number, an operator, a group, a function, an axiom, a convention-and any one of these may have special properties attached or particular limitations 
imposed. And when to this are added many other symbols with similar extensions of meaning, one difficulty of mastering a memoir of average length becomes sufficiently obvious. The language must first be learned, and a new language in general confronts the reader when he turns to some memoir by a different author, even on the same subject.

Is there a remedy for this condition? Can mathematical notation be standardized to some extent and if so, can it be done in such a manner that it will meet with general acceptance and use? It is perhaps a Herculean task but I am not at all sure that a concerted effort would not result in some improvement.

With what material have we to deal? On the one side there are the four double fonts of the Roman, Italic, Greek, and German alphabets, one or two fonts of the Arabic numbers and a few well-known symbols, the types for which exist in any good printer's composing room. On the other side there is a considerable body of ideas to be represented, although the number of those most frequently used is not very great. Suppose, by way of illustration, that some very general conventions were agreed upon: for example, that operators should be denoted by capital letters, except in the few cases like those of differentiation or integration, where the notation is already standardized; continuously varying quantities by certain groups of lower case letters, discontinuous quantities like those in our number system by other groups, quantities remaining fixed by still other groups. Continuing with this idea we should represent those properties or limitations which are of frequent occurrence by certain combinations of marks and letters and it would be advisable to agree that the older signs, those of the four chief arithmetical operations for instance, shall retain their original meanings if unattended by any distinguishing mark. Any such plan should depart as little as possible from the schemes of notation which have been most generally used. One result undoubtedly would be some loss of the brevity which now characterizes the mathematical symbolism of much of the published work. I doubt, however, whether this would be an evil, in fact, it might be an advantage; the reader would soon become familiar with many groups of symbols and would read their meaning without effort, just as he recognizes a printed word not by the separate letters but by the appearance of the group of letters which form the 
word. After all, brevity is not the soul of mathematics. If a scheme with such broad outlines is not feasible, it might still be possible to apply the method separately to divisions of mathematics. But this idea is less attractive and presents additional difficulties in the overlapping of different divisions. Most of us would prefer, I think, to learn one language with many words, than several different languages even though their vocabularies were much smaller.

It may be objected that any standardization of symbolism may seriously interfere with the freedom of the investigator and so limit his output. One may ask in reply whether past liberty has not already degenerated into license and whether some regulation will not confer benefits on mathematics similar to those we acknowledge in the framing of laws for the welfare of the community. If the circulation of the output be increased we can well afford some decrease in the quantity.

It is a much more simple matter to suggest a method for bringing any such scheme or schemes into operation if once agreed on by an authoritative committee. After proper and frequent publication, there is no need for any kind of compulsion; a writer, using a settled scheme has merely to say so. In this way it would either come into general use or die a natural death without having caused any particular harm. There is another consideration in this connection. We must look forward to the time when our turn will come to publish a revised encyclopædia of mathematical sciences; if standards of notation are then available, one can hardly overemphasize their value in making a summary of the work of the past.

In the meantime some assistance can be given by such aids as tables of notation and separate summaries attached to each paper published, even at the cost of artistic appearance; this has already become a feature of some journals in other subjects. And there are probably other devices which might usefully be employed to assist the reader.

These are, however, mechanical aids and only touch on the fringe of the question which is my principal theme-the isolation of mathematics from the natural sciences. There is, I must confess, at present little prospect of the cure generally proposed, namely that mathematicians turn their attention to the applications. And there is good reason why this is not acceptable, at least in the form usually stated. To make progress in any one direction requires a more considerable 
knowledge of the physical laws and past achievements in that direction than most of those who are making advances in their own lines have time to acquire. They may do much by encouraging a certain proportion of the students who come under their influence to attack such problems. The welcome given by the mathematical journals in America to memoirs on the applications is a sufficient answer to those who would believe that the study of the latter is discouraged. In this connection it is noticeable that the pure science has a tendency to drive out the applied science-this has been peculiarly evident in England during the last quarter of a century - or to put the matter in another form, the cleavage between pure mathematics and the experimental sciences which use mathematics has tended to increase.

It is necessary here to distinguish between the study of the mathematical methods used in the applications and the study of the applications by mathematical methods. In the former, the foundation, the logic and the limitations which must be imposed on the processes adopted by applied mathematicians are considered; in the latter, it is new phenomena or the coordination of known phenomena which are sought. Poincaré, who produced much in both directions, obviously kept the distinction clear in his own mind, even when he mingled the two in a single memoir like his treatise on celestial mechanics. And his point of view in the former was not that of criticism but of justification of the methods used in the latter. Unfortunately, while a study of methods clarifies and expounds, it is not as a rule fertile in producing new advances. It is only when the mathematician leaves aside the particular applications of a method and studies the latter in all its bearings that he is able to obtain novelty.

The study of physical laws and phenomena by the methods of mathematics stands on an altogether different basis. Why is it that with calls in every direction for help so little is done? One hears blame cast on the mathematician for this state of affairs and perhaps to some extent there is justification for it. But I believe that the blame must be shared at least equally by the physicist or engineer who asks for the solution of his problems. The latter are generally presented in such a manner that the mathematician who is not thoroughly familiar with the subject cannot even make a beginning; often he is not given the necessary information about the physical cir- 
cumstances which will enable him to formulate the problem. In its generality, it may be quite beyond the present state of mathematics to give a solution and yet if he is allowed to neglect certain factors and to modify others it may be possible to obtain the main facts needed. But he cannot be expected to know all these details. The more difficult the problem, the more necessary is a full and complete statement of everything connected with it. The physical laws which may be assumed and which cover the principal features, the modifying circumstances which may be left aside if the problem is too difficult, the range of numerical values which constitute the data, the range of numerical values which are needed in the solution-all these should be carefully set forth in any statement of the problem.

It is this work of formulation that is mainly contained in the treatises on applied mathematics so far as the older and more simple problems suggested by natural phenomena are concerned. They also give the principles which should be followed in all cases. Thus the knowledge of the applied mathematician, using the word in the old sense, is necessary as the intermediary between the experimentalist and the pure mathematician if one is to help the other. He must have a considerable knowledge of the phenomena and with it a sufficient knowledge of mathematics; neither can be neglected. It is the almost complete disappearance of this type of scholar from among us that is perhaps responsible more than anything else for the isolation of which I have spoken. The temptation either to proceed to experiment or to turn to purely mathematical researches has been too strong.

The need of a remedy is obvious and in searching for it we may perhaps have to consider a change in our system of regarding the doctor's degree with its original thesis as a necessary preliminary to every opening which leads to scientific work. It is frequently stated that the main difficulty in obtaining applied mathematicians is the difficulty of finding a suitable thesis for a student, by an instructor who has not himself investigated in those lines, and we have few here who have done so. This is unquestionably true. Under the circumstances, those organizations which need such men for their own purposes, whether they be government bureaus or commercial houses, must create positions for applied mathematicians with the same advantages that they give to those who 
perform experimental researches. They must understand that the type of man needed must be trained for the purpose, that he cannot usually be solely a physicist or solely a mathematician but must have received a sufficient education in both directions. If the demand is clearly set forth, there is little doubt that the supply will be forthcoming.

The necessary link once supplied, the pure mathematician can render valuable services. But to do so, he will need to change to some extent the methods which he is accustomed to employ. The generality and completeness which he is accustomed to give to his researches are rarely useful in the applications. What is needed is the discovery of solutions which in a limited range can be reduced to numerical values without an excessive amount of labor. Singularities, for example, are usually excluded for one cause or another; places where the function is regular are those commonly needed. If the constants expressed by symbols are so numerous that the problem is intractable, some of them must be given numerical values; it will frequently happen that all needed information can be obtained from two or three well chosen numerical cases. And it is not only the material stored up in the past that will be used; new devices will continually be needed and I do not think that he will find the problems any less worthy of his best efforts than those to which he has been in the habit of directing his attention. The chief difference will perhaps consist in a limitation to work in definite directions instead of roaming in the field of discovery with freedom to investigate wherever the prospect seems to attract.

It follows that some organization of the different processes needed in the applications of mathematics has become necessary. The simple problems and a very few of the difficult ones have been solved and progress is halted in the face of a demand which is greater than ever before. Whether one considers questions of purely scientific interest like those which are presented in celestial mechanics or questions of practical importance, it is still true that there are few workers and even with their combined efforts they cannot achieve much. If we are to enlist the services of others, it is necessary that the work be divided in such a manner that contributions to its various parts can be made by those who have the proper training and qualifications. The first question suggested by what has been said is to ask what part can be taken by those who have a training in pure mathematics. 
It is always safe but not always useful to restrict oneself to generalities. I am fully aware of the danger which attaches to the mention of specific problems; they have been solved, or they are insoluble with known methods, or they are not suitable for mathematical treatment. Nevertheless, I am going to mention briefly two or three which I believe cannot properly be placed in any one of these excluded classes. So far as I know, there is no published systematic attempt to treat them in such a manner as to furnish results in a form available for actual application. Too much emphasis can scarcely be laid on the last point if the researches of mathematicians are to be connected with the applications. Much of such work may be tedious and uninteresting but there is also much which demands the exercise of the best powers which an investigator may possess. He is also in the most favorable position to do it. $\mathrm{He}$ has usually looked at his subject from many directions before setting forth that one which is best suited for a theoretical exposition and he will know and be able to develop most easily that which is suitable for the applications.

Of the fundamental requisites which need systematic examination, perhaps that of most practical value is an inquiry into the symbolic forms and modes of expression which are best adapted for numerical calculation. On the one side there exists a wealth of material in the shape of formulas, theorems, and methods available for choice. On the other side the machinery to which these are to be adapted is limited. Practically, the only numerical operations which can be performed mentally are addition, with its counterpart subtraction, and those operations in which a knowledge of order of succession in our decimal number system can be of assistance. All other operations are performed by means of tables whether memorized like the multiplication table or printed. Mechanical and graphical devices to replace these are available but the only one which has complete accuracy is that which replaces the mental operations, namely, the adding machine. The number of printed tables at our disposal is so small that a classification of these containing an account of their contents and of the uses to which they may be put is not a serious undertaking as a first step. Finally, the various devices by which the labor of numerical computation may be diminished should be collected; this is more troublesome because many of them are the results of experience and either have not been 
published or are only mentioned incidentally in connection with special investigations.

It is a much less simple matter to try to point out a method of procedure for the adaptation of symbolic forms so that full use may be made of this numerical machinery. Something might certainly be achieved by gathering together those forms in most common use in a given subject or group of subjects and making a study of them from this point of view. An example occurs in the case of Fourier series; a number of papers have been published during the present century on the methods for connecting a Fourier series with its numerical values although most of the attention given has been in the direction of analysis rather than synthesis except in the case of purely mechanical devices. One may perhaps look forward to the construction of a more general theory which shall have as its object the formulation of laws or rules which will serve in all cases. Adaptation to numerical computation and the performing of the computations themselves are by no means the simple matters which they are frequently assumed to be if they are to be efficiently done. And it is this efficiency which must be the goal.

The converse problem of reducing a series of numerical values which obviously obey some law to symbolic form is equally in need of systematic treatment. If the law is known or there are reasons for the assumption of a specified form, the method of least squares enables the unknown constants to be determined. But it is rare that one opens a series of memoirs containing investigations in the physical sciences without seeing diagrams for which no law is known; in many cases no attempt is made to find one. The author has perhaps drawn a smooth curve which he judges would most nearly represent the facts if the observations had been freed from the errors which are incidental to all experimental work. But progress is halted until the curve can be so expressed that the relation of the physical phenomena that it represents to other phenomena or to known physical laws can be obtained. The best method of doing this is usually through a symbolic formula. The mere fact that functions with limited variation (and the known phenomena possess this property) can be represented by a Fourier series is usually of little or no assistance. Even if it be so represented it conveys almost no further information unless the phenomena themselves are of a periodic nature. 
The Fourier series might perhaps be used as a first step in the investigation but there arises the difficult and practically unsolved problem of finding the function represented by the Fourier series, as was pointed out in a recent address. Some more direct and practical method is needed. In certain cases a mode of procedure can be indicated. When the curve appears to resemble the graph of some known function, it should be possible to devise methods of approximation by which additions to or changes in the function can be obtained, the constants present being then determined by the method of least squares. Transformations of functions so obtained involve the study of forms numerically equivalent within a given range to a given degree of accuracy.

The case of periodic or quasi-periodic functions deserves special mention. Light-curves of many stars are now being obtained with considerable accuracy. Some of the curves differ from a simple sine-curve so greatly that the representation by Fourier series will probably involve the presence of a large number of terms with sensible coefficients. Such forms will probably require transformation into other periodic functions if any clue is to be obtained to the laws which govern these changes. Our own sun is no exception. We obviously have to deal with forces which do not produce regular periodicity, but until some analytic forms can be obtained which will represent the curves with sufficient brevity and accuracy there is little hope of unravelling their mysteries.

I have mentioned these problems particularly because they lack the definiteness in the results demanded which characterizes most of the usual problems of mathematics. I do not think they belong to the subject less for that reason. Mathematics certainly can lay claim among its other properties to be a method of organizing ideas, and it has effectively done so in the past. The result of tossing a coin into the air is, owing to our lack of ability to work out all the consequences of the laws of mechanics, quite as indefinite, and yet mathematics has built up the solid and definite set of rules which we know as the theory of probabilities - a theory which is in practical use in every insurance company and in all scientific work: it is difficult to imagine what modern astronomy would be today without the method of least squares. Such problems as I have suggested may possibly have only temporary value but if in their solution some order is produced from chaos, a 
contribution of practical use, at least in our time, will have been made.

Perhaps the set of mathematical problems most important for the applied sciences are those included in obtaining numerical solutions of differential equations. In many cases the ordinary methods such as finding exact solutions in terms of known functions or by means of series fail. The failure in the latter case is not a mathematical failure, but a practical one, owing to the large amount of numerical computation needed. Where it has to be undertaken, the particular method of analytical continuation of series, known as mechanical quadratures, is adopted. This certainly achieves the object in view but usually with a lack of real efficiency which cannot fail to strike anyone who attempts to use it to any extent. And it naturally becomes the more troublesome whenever the curve has any special feature such as near approach to a singularity. Questions which seem unimportant in theory sometimes loom large in calculation. For example, how many places of decimals at the start and throughout the work are needed to secure a given standard of accuracy? Is the same degree of accuracy necessary throughout? How large may each arc be taken? And so on.

If one examines textbooks or treatises for information, it is difficult to find anything of real use; in fact, the method as applied to the solution of, for example, a pair of ordinary simultaneous differential equations, I have not seen mentioned. In the form given in the investigations where it has been used, it is doubtless capable of great improvement. We may further ask: under what forms can it be used so that each arc shall be as long as possible with the same degree of accuracy? Is it necessary to confine it to power series and can it not be developed for computation in series of other functions so that, in any special case, one shall have a choice which can be made according to circumstances laid down in advance? Unquestionably there is material at hand to answer some of these questions if it could be worked up into a form simple enough for a person not possessing an intricate knowledge of differential equations and the properties of various modes of expansions to use. I hesitate in mentioning solutions of partial differential equations in this connection on account of the many inherent difficulties. Nevertheless the new problems arising in aviation and the older set connected with the motions 
of our atmosphere and the prediction of the weather perhaps transcend in importance all others.

These problems are fundamental in the applications of mathematics. The expert knowledge and insight of the student of pure mathematics would seem to point him out as the one most likely to achieve at least partial successs in dealing with them. At the same time, little or no knowledge of physical processes and results is required. But if he happens to possess special information in any one of the natural sciences, I have little doubt that he can find a field giving a stimulus for new work and an opportunity to connect his subject with the outside world. Even biology is now demanding help in certain directions, somewhat reluctantly it is true, but none the less surely. Even if mathematics cannot always assist in solving problems, it can still help through its advantages as a brief and clear mode of description where processes are complicated and interdependent. But whatever is done in any direction, the work will be of little practical value unless it satisfies the final test, namely, that the form in which it is left is such that it may be applied without a full knowledge of the processes by which it is reached.

It is perhaps easy for one who has spent half his life in an undertaking which must always have numerical results as the final test, to exaggerate the importance of all work of this nature. This particular problem, it is true, has a variety which is not common to many such undertakings. Perhaps the most unexpected result has been that nearly all the portions which can be said to possess some small degree of novelty have arisen from the necessity of obtaining formulas which should give numerical results more easily than the older developments. And it has not infrequently happened that the actual computation of a particular case has indicated immediately the method which should be followed in the general case. This fact may not be a recommendation to the person who has the desire to use his powers to their fullest extent, yet for one who has a definite end in view and who wishes to reach the goal by the shortest route it may appeal as having certain advantages. Moreover, the time spent in actual computation is usually not a large fraction of that necessary for the whole work; if there are many computations of the same nature, each one may take on the average only a fifth or a tenth of the 
time that an isolated case would require. The construction of numerical tables is a striking example of this as soon as the work has been properly organized.

Much of the mathematics used in the applications is repulsive to the pure mathematician, and properly so. It lacks the form and symmetry which so often assist in showing the ideas which are in process of development. There is a good reason for this. For efficient numerical application, many properties possessed by the initial formula and particularly that of symmetry should be used to assist; in the process of transformation they must necessarily disappear. The limitations, too, which are often of greater interest, are usually so much broader in the mathematics than in the applications demanded that there is no need to examine them. Or if they are reached, the numerical work at once warns of their approach and the formula or method is changed. Unfortunately, too, the very devices which constitute a good formula or by which a solution is reached often admit of such simple mathematical description that the air of mystery disappears and there is little external evidence of any special ingenuity. Nevertheless there is a degree of mental satisfaction in the achievement of concrete results which is a sufficient reward to those who work for them.

In this attempt to emphasize the need for more attention to the applications of mathematics, it is hardly necessary for me to deny a possible implication that the further study of the pure science is unprofitable. On the contrary, there seems to be more need for it than ever. But I believe that this need demands studies which shall take directions more immediately helpful to other sciences, not only for their assistance but for the sake of the subject itself. We cannot leave such matters to the chances of the future; some definite effort is required to work towards a goal which has been neglected in the past. It demands, it is true, limitations on one's freedom to follow attractive openings, it may require tedious and uninteresting developments, but these will not be deterrents to anyone who has previously attempted to advance knowledge without hope of external reward. Moreover, the natural bent of a nation which has been compelled by its environment to regard most of its affairs from a thoroughly practical point of view, gives promise of success. An incentive and the training once supplied, there seems to be no reason why a school of applied 
mathematics of the first rank should not flourish on this continent.

Finally, any discussion which has investigation as its theme is incomplete if it leaves out of consideration the events which are taking place among the older nations. Although as a body and as individuals we have perhaps been less affected than others engaged in intellectual pursuits, nevertheless, these events cannot fail now and more so in the future to modify -perhaps to change entirely-our outlook. While the need for scientific research has been emphasized and brought forward more strongly than ever before, and in this movement we hope to share, yet it is to be remembered that a practical end has been visible throughout. Little stress has been laid on its purely intellectual side and we, in common with others engaged in research in pure science, may fear neglect and increasing lack of support. For a time at least, the problems of reconstruction and adaptation to new conditions will demand of the older nations most of the time and energy which has in the past been allowed to run in less practical routes. It is true that here we are happily removed from the devastation which has overtaken Europe, but the lack of stimulus from abroad cannot fail to have its effect. We need at this time to take stock of the resources of ourselves and of our Society, both mental and physical, to find whether they are sufficient for what may come and more than all to conserve the heritage which we have received from the old world so that it may later be returned alive and productive.

The possibility of great changes coming rapidly and with little warning must be faced. We cannot therefore be content to sit back and await events. The future of research in pure science is in danger as never before and the less practical the science the greater the danger. New economic and social forces are at work in every direction and may call many of us to activities which may stop temporarily and perhaps permanently the work and thought which have in the past most fully occupied us. Two generations of steady and continuous development have tended to make us settle in grooves of thought and habits of mind in which great upheavals have no place. To such changes at least a proper mental attitude is necessary. Action may not be needed now but preparation for action is essential and the chances of the future should be in the mind of every individual if the right course is to be pursued 
when the time for action comes. Even at the present moment we can no longer regard the Society merely as one to forward mathematical research in America; the wider interests of the subject have now to be considered, since we form the one large national organization devoted to research in mathematics which can continue its work unhindered by more pressing claims. In these wider interests our opportunities for service may come: the readiness and ability that we show in a realization and fulfilment of them will be the chief measure of our success in the past and promise for the future.

YALE UNIVERSITY, New Haven, Conn.

\title{
A THEOREM ON THE CURVES DESCRIBED BY A SPHERICAL PENDULUM.
}

\author{
BY PROFESSOR ARNOLD EMCH.
}

(Read before the American Mathematical Society, December 27, 1916.)

1. IN what follows I shall restrict myself to the case considered by Greenhill,* in which the pendulum bob reaches (but does not go above) the horizontal plane of suspension with a non-vanishing horizontal velocity. $\dagger$

When this assumption is made, there exists an interesting relation between the curve described by the bob and a certain quartic in space. It is the purpose of this note to establish this simple relation.

2 . Let $r_{0}$ be the distance of the bob from the vertical axis when its horizontal projection crosses the $x$-axis; $A+2 \pi$ the horizontal angle between two consecutive positions in the plane of suspension; $4 K$ the real period in the Jacobian elliptic functions appearing in the solution; $v=t / 2 w_{1}$, where $w_{1}$ is the real half-period in the Weierstrassian form, and $t$ the time; $a, b, c(a>b>c)$ the real roots of the cubic in the general solution; $\mu=\sqrt{\left(l^{2}+a^{2}\right) /\left(l^{2}-a^{2}\right)}$, where $l$ is the radius of

* Greenhill, Les Fonctions elliptiques et leurs Applications, chap. III. Appell, Traité de Mécanique rationnelle, t. 1, p. 494. Tannery et Molk, Eléments de la Théorie des Fonctions elliptiques, t. 4, p. 188.

$\uparrow$ The statement by L. Lévy, on page 161 of his Fonctions elliptiques, that in this case $x, y, z$ are doubly periodic functions of the time is not correct. 